OPEN ACCESS

Edited by:

Rashmi Kumar,

King George's Medical University,

India

Reviewed by:

Prashant Jauhari,

All India Institute of Medical Sciences,

India

Patricia Rzezak

University of São Paulo, Brazil

*Correspondence:

Syuan-Yu Hong

dazingdog@hotmail.com

Specialty section:

This article was submitted to

Pediatric Neurology,

a section of the journal

Frontiers in Neurology

Received: 13 December 2018

Accepted: 02 May 2019

Published: 15 May 2019

Citation:

Lin C-H, Lin W-D, Chou I-C, Lee I-C and Hong S-Y (2019) Epilepsy and

Neurodevelopmental Outcomes in Children With Etiologically Diagnosed Central Nervous System Infections: A

Retrospective Cohort Study.

Front. Neurol. 10:528.

doi: 10.3389/fneur.2019.00528

\section{Epilepsy and Neurodevelopmental Outcomes in Children With Etiologically Diagnosed Central Nervous System Infections: A Retrospective Cohort Study}

\author{
Chien-Heng Lin ${ }^{1,2}$, Wei-De Lin ${ }^{3}$, I-Ching Chou ${ }^{4,5}$, Inn-Chi Lee ${ }^{6,7}$ and Syuan-Yu Hong ${ }^{4 *}$ \\ 'Division of Pediatrics Pulmonology, China Medical University, Children's Hospital, Taichung, Taiwan, ${ }^{2}$ Department of \\ Biomedical Imaging and Radiological Science, China Medical University, Taichung, Taiwan, ${ }^{3}$ Department of Medical \\ Research, China Medical University Hospital, Taichung, Taiwan, ${ }^{4}$ Division of Pediatrics Neurology, China Medical University, \\ Children's Hospital, Taichung, Taiwan, ${ }^{5}$ College of Chinese Medicine, Graduate Institute of Integrated Medicine, China \\ Medical University, Taichung, Taiwan, ${ }^{6}$ Department of Pediatrics, Chung Shan Medical University Hospital, Taichung, Taiwan, \\ ${ }^{7}$ School of Medicine, Institute of Medicine, Chung Shan Medical University, Taichung, Taiwan
}

Background: Central nervous system (CNS) infection in childhood can lead to neurological sequelae, including epilepsy, and neurodevelopmental disorders, such as attention-deficit/hyperactivity disorder (ADHD) and autism spectrum disorder (ASD). This study investigated the association of etiologically diagnosed childhood brain infections with the subsequent risks of epilepsy and neurodevelopmental disorders.

Objectives: We retrospectively analyzed the data of children aged $<18$ years who had definite brain infections with positive cerebrospinal fluid cultures from January 1, 2005, to December 31, 2017. These patients were followed to evaluate the risks of epilepsy and neurodevelopmental disease (ADHD and ASD) after brain infections (group 1) in comparison with the risks in those without brain infections (group 2).

Results: A total of 145 patients with an average age of 41.2 months were included in group 1. Enterovirus accounted for the majority of infections, followed by group B Streptococcus, S. pneumoniae, and herpes simplex virus. A total of 292 patients with an average age of 44.8 months were included in group 2. The 12-year risk of epilepsy in group 1 was 10.7 (95\% confidence interval [Cl], 2.30-49; $p<0.01$ ). Compared with group 2 (reference), the risk of ASD in the age interval of 2-5 years in group 1 was 21.3 (95\% Cl, 1.33-341.4; $p=0.03)$. The incidence of ADHD in group 1 was not significantly higher than that in group 2.

Conclusions: This study identified the common etiological causes of brain infections in Taiwanese children. The highest-risk neurodevelopmental sequelae associated with brain infections was epilepsy. Children who had a diagnosis of brain infection (specially Enterovirus) should be followed since they are at greater risk of developing epilepsy and ASD.

Keywords: epilepsy, neurodevelopmental outcomes, central nervous system infections, brain infections, children 


\section{INTRODUCTION}

Central nervous system (CNS) infections in childhood can be devastating and may lead to neurological sequelae or death. However, different pathogens imply different neurological prognoses. A greater understanding of causative agents may lead to greater opportunities to predict associated neurological outcomes of children later in life (1). The risk of unprovoked seizures after encephalitis and meningitis has been explored in a previous study (2). Although seizures occur frequently in children with acute bacterial meningitis, only those with permanent neurologic deficits after meningitis are at a high risk of epilepsy $(3,4)$. Seizures also occur in children with acute viral encephalitis and increase the risk of later unprovoked seizures and epilepsy (5).

Neurodevelopmental disorders (NDDs) are a group of conditions that interfere with CNS development at an early age. Developmental brain dysfunction causes later neuropsychiatric problems that persist into adulthood. There are various types of NDDs; common NDDs in children are attention-deficit/hyperactivity disorder (ADHD), autism spectrum disorder (ASD), learning disabilities, and intellectual disability (6).

The pathogenesis of epilepsy and NDDs in children has not been completely understood. It is generally known that they are caused by several factors, including genetic factors, neurobiological factors, environmental and perinatal factors, parental age, and maternal medication use during pregnancy (7). Among them, CNS infections play a pivotal role as environmental and perinatal factors (8).

The impact of CNS infection in childhood is considerably different from that in adulthood. Children acquire CNS infections through various routes and at different time points. Maternal infections during pregnancy, regardless of viral, bacterial, or parasitic illnesses, have been shown to be associated with fetal brain malformations, including intracranial calcifications, cortical atrophy, microcephaly, and hydrocephalus. Simultaneously, the inflammatory pathways caused by infections lead to the release of various inflammatory cytokines and morphological changes consistent with an infectious intrauterine environment or umbilical cord, causing the immature CNS to be at risk (9). When newborn infants move through an infected birth canal, they are susceptible to encephalitis, especially that caused by HSV and GBS. Neurological dysfunction associated with brain infections during CNS development often exceeds cellular damage directly attributable to virus replication and thus results in permanent neurological deficits (10). In addition, neurologic symptoms of CNS infection in young children can be subtle and indolent and can easily be missed owing to the lack of classic adult symptoms (11). When CNS infection diagnosis is delayed in an infant or child, the consequences can be harmful. Some evidence suggests that brain infections in infancy or childhood compromises early CNS development and might increase the risk of epilepsy and other NDDs later in life (12-15).

This study explored the neurodevelopmental outcomes of childhood brain infections with different microbiological etiologies, with the aim of developing strategies of prevention, early diagnosis, and targeted treatments for epilepsy and NDDs after CNS infections in children.

\section{MATERIALS AND METHODS}

In this retrospective cohort study, we analyzed patients $<18$ years with clinically suspected CNS infections and collected the data of them who had definite brain infections with positive cerebrospinal fluid (CSF) cultures between January 1, 2005, and December 31, 2017. The preliminary inclusion protocols based upon the following criteria (16):

Altered mental status lasting $\geq 24 \mathrm{~h}$ with no alternative cause identified, plus $\geq 2$ of the following for a "possible" diagnosis or $\geq 3$ of the following for a "probable" diagnosis:

1. Documented fever $\geq 38^{\circ} \mathrm{C}\left(100.4^{\circ} \mathrm{F}\right)$ within $72 \mathrm{~h}$ (before or after) presentation.

2. Generalized or partial seizures not fully attributable to preexisting seizure disorder.

3. New onset focal neurologic findings.

4. CSF WBC count $\geq 5$ cells/microL.

5. Abnormality of brain parenchyma on neuroimaging suggestive of encephalitis that is new or appears to have acute onset.

6. Abnormality on EEG that is consistent with encephalitis and not attributable to another cause.

A comprehensive medical record review was strictly enforced to exclude the children who had either epilepsy, neurologic, metabolic, autoimmune, or any other congenital disorders before the onset of CNS infection. Individuals who had more than one episode of CNS infection were also excluded from the final analysis. The last subject was enrolled in December 2017. All patients included in the study (study and controls) had a follow-up period began from baseline (the time point they were included in the study) until the end of follow-up (October 30, 2018); or death. We conducted the follow-up measures through medical records, telephone or e-mail access to the families quarterly. Once a suspected NDD was noticed, we contacted the children returning to our Pediatric neurology clinic for a comprehensive assessment.

To focus on bacteria and certain viruses that can be cultured from CSF, we sent patient's CSF for Gram stain, bacterial culture and viral studies (viral culture, polymerase chain reaction).

A positive CSF microbial etiology was defined as one yielding pathogens from CSF through multiplex polymerase chain reaction testing and viral and bacterial culture (17). To determine the key infectious etiology, patients with more than one pathogen isolated from CSF underwent the following to obtain more clinical information: the culture of other sites or CSF and serum antibody testing. After the screening process, the final study population comprised 145 subjects. Among the study subjects, we also looked into the issues during their admission which may be related to long-term neurological sequelae: With or without ventilator use; 1st day Pediatric Glasgow Coma Scale, seizures pattern (if any); type of Brain infections (by analyzing their 


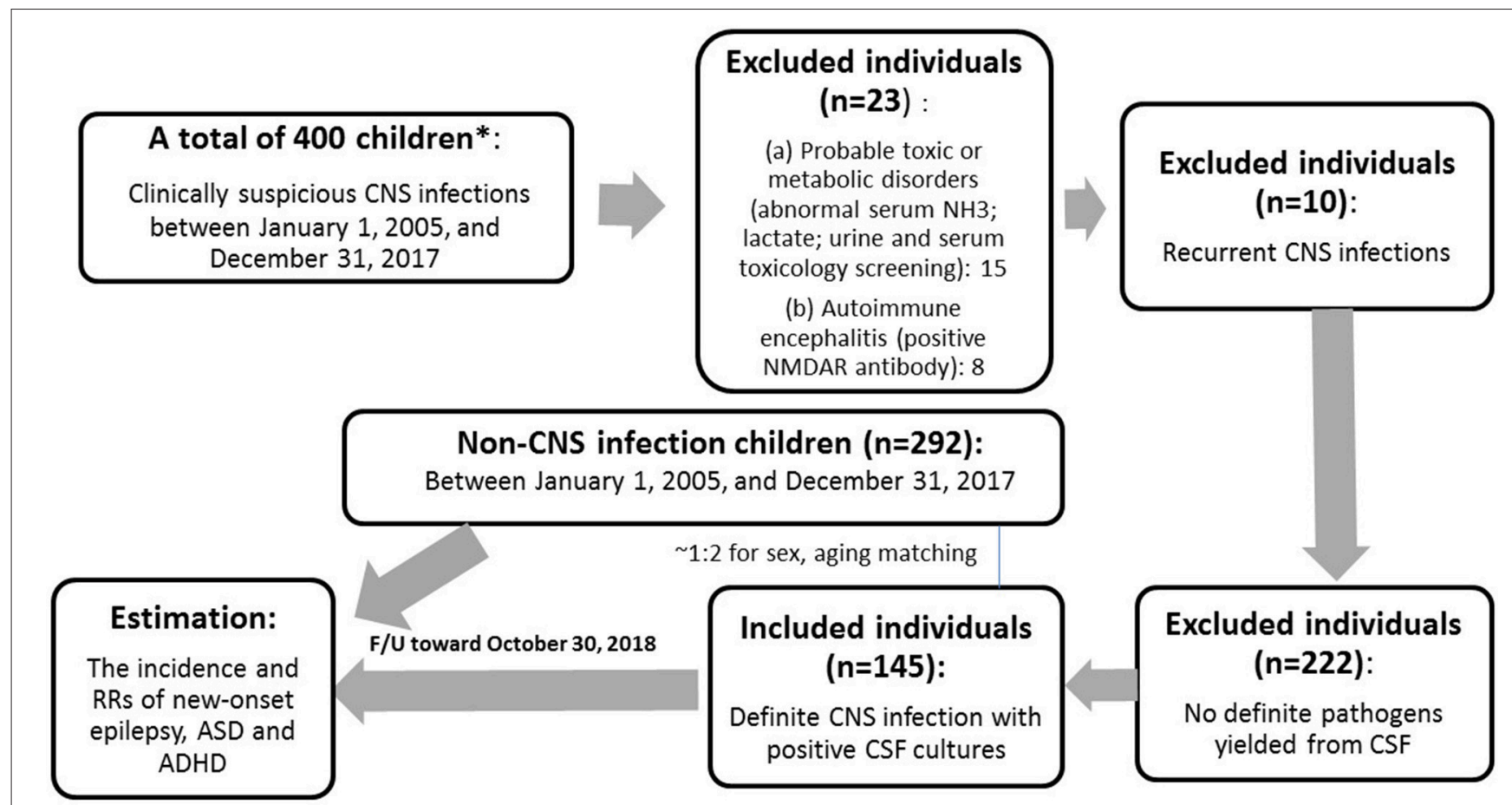

FIGURE 1 | Flowchart of the study. *The patients who were screened from the medical records and contacted by the our case managers.

neuroimaging or by clinical judgment of individual physicians), with a view to see the association between various brain infections (meningitis, encephalitis, abscess, etc.) and clinical risk factors at admission with subsequent NDDs.

Instruments used in the assessment of children and adolescents with suspected intellectual disability (ID) were: Bayley Scales of Infant and Toddler Development, 3rd Edition (for young children $<2$ years), Wechsler Preschool and Primary Scale of Intelligence (for children ages 2 years 6 months to 7 years 7 months) and Wechsler Intelligence Scales for Children, 5th Edition (WISC-V) (for children in school age and beyond). Epilepsy (our outcome of interest) was defined as 2 unprovoked seizures more than $24 \mathrm{~h}$ apart whose diagnosis was made by a Pediatric neurologist. Patients who met the relevant diagnostic criteria in the Fourth and Fifth Editions of Diagnostic and Statistical Manual of Mental Disorders were diagnosed with ASD and ADHD, and their diagnosis was made by a Pediatric psychiatrist or Pediatric neurologist in the inpatient or outpatient setting of China Medical University Children's Hospital between January 1, 2005, and December 31, 2017. We selected 292 children with unspecified infections (acute gastroenteritis, acute tonsillitis, urinary tract infection, and others) between January 1, 2005, and December 31, 2017, whose positive culture results were yielded from throat, urine, stool, or other body discharges but not from blood or CSF as a matched control group in terms of age and mean follow-up years. The control group was derived from the outpatient clinic and had been confirmed to have fully recovered from their mild illness during follow-up (Figure 1).
Gender, age of infection onset, and associated pathogens constituted confounding factors. The study population was divided into 4 groups based on the age of infection onset: ages 0-2 years (infants and toddlers), ages 2-5 years (preschoolers), ages 5-10 years (young children), and ages $>10$ years (young teens and teenagers) (Table 1). The study protocol was approved by the Ethics Review Board of the China Medical University Ethics Committee. Categorical variables between groups were analyzed using $\chi^{2}$ tests. Moreover, we calculated the incidence density rate of epilepsy, ADHD, and ASD in the control group and the brain infections group (which was subdivided into different age intervals and subdivided by pathogens). Using risk regression model, we estimated of the risk ratios (RRs) and 95\% confidence intervals (CIs) of epilepsy, ADHD, and ASD in the brain infections group relative to the control group. All statistical analyses were performed using PASW Statistics version 18.0 software (SPSS Inc., Chicago, IL, USA). In addition, for all executed statistical analyses, we deemed 2-tailed $p<0.05$ to indicate statistically significant results.

\section{RESULTS}

\section{Data Analysis}

From January 1, 2005, to December 31, 2017, a total of 145 children who were diagnosed with brain infections with definite pathogens and 292 children with non- brain infections were enrolled into this study. Table 1 presents the participants' demographic factors. The participants' mean age was 3.43 years (standard deviation [SD], 5.05). The proportion of boys was 
TABLE 1 | Comparison of demographics between brain infections and controls.

\begin{tabular}{|c|c|c|c|}
\hline & \multicolumn{2}{|c|}{ Group } & \multirow[t]{2}{*}{$p$} \\
\hline & $\begin{array}{l}\text { Brain infections } \\
(N=145, \%)\end{array}$ & $\begin{array}{c}\text { Controls } \\
(N=292, \%)\end{array}$ & \\
\hline $\begin{array}{l}\text { Mean follow-up years } \\
\text { to epilepsy (SD)* }\end{array}$ & 7.7 (3.6) & 7.9 (3.5) & $>0.99$ \\
\hline $\begin{array}{l}\text { Mean follow-up years } \\
\text { to } A D H D(S D)^{*}\end{array}$ & $7.6(3.8)$ & $7.8(3.6)$ & $>0.99$ \\
\hline $\begin{array}{l}\text { Mean follow-up years } \\
\text { to } A S D(S D)^{\star}\end{array}$ & $7.7(3.8)$ & $7.9(3.7)$ & $>0.99$ \\
\hline Gender & & & 0.28 \\
\hline Male & $98(67)$ & $182(62)$ & \\
\hline Female & $47(33)$ & $110(38)$ & \\
\hline $\begin{array}{l}\text { Infection mean age } \\
\text { (months) (SD) }\end{array}$ & $41.2(60.70)$ & $44.8(41.24)$ & 0.46 \\
\hline Stratified by age (yr) & & & $>0.99$ \\
\hline $0-2$ & $92(63.4)$ & $184(63)$ & \\
\hline $2-5$ & 15 (10.3) & $31(10)$ & \\
\hline $5-10$ & $16(11.0)$ & $33(11)$ & \\
\hline$>10$ & $22(15.1)$ & $44(15)$ & \\
\hline Associated pathogens & & & N/A \\
\hline Enterovirus & $78(53.7)$ & - & \\
\hline Herpes simplex virus & $8(5.5)$ & - & \\
\hline $\begin{array}{c}\text { Group B } \\
\text { streptococcus }\end{array}$ & 28 (19.3) & - & \\
\hline S. pneumoniae & $10(6.8)$ & - & \\
\hline Others & $21(14.4)$ & - & \\
\hline $\begin{array}{l}\text { Neurodevelopmental } \\
\text { outcomes }\end{array}$ & & & $<0.01$ \\
\hline ID or CP & $48(33.1)$ & $9(3.0)$ & \\
\hline Epilepsy & $11(7.5)$ & $1(0.3)$ & \\
\hline ADHD & $4(2.7)$ & $6(2.0)$ & \\
\hline ASD & $1(0.7)$ & $0(0)$ & \\
\hline Epilepsy+ADHD & $1(0.7)$ & $1(0.3)$ & \\
\hline $\mathrm{ADHD}+\mathrm{ASD}$ & $1(0.7)$ & $1(0.3)$ & \\
\hline
\end{tabular}

${ }^{*} t$ test.

higher than that of girls (67 vs. $33 \%$ ). The mean (SD) follow-up years for participants with epilepsy, ADHD, and ASD were 7.7 (3.6), 7.6 (3.8), and 7.7 (3.8), respectively. The minimal follow-up duration was 11 months year since the last subject was included in December 2017 and the maximum follow-up duration was 13 years and 10 months since the first subject was included in January, 2005.

The common pathogens of brain infections in this study were enteroviruses $(n=78,53.7 \%)$, herpes simplex virus (HSV) $(n=8,5.5 \%)$, group B Streptococcus (GBS) $(n=$ $28,19.3 \%)$, Streptococcus pneumoniae $(n=10,6.8 \%)$, and others $(n=21,14.4 \%)$ - namely, Adenovirus $(n=2)$, HHV$6(n=2)$, HHV-7 $(n=1)$, Parvovirus $(n=1)$, Varicellazoster virus $(n=1)$, Citrobacter koseri $(n=1)$, Enterobacter hormaechei $(n=1)$, Enterococcus casseliflavus $(n=1)$, Escherichia coli $(n=2)$, Haemophilus influenzae $(n=2)$, Neisseria meningitidis $(n=1)$, Listeria monocytogenes $(n=$
1), Mycobacteria tuberculosis complex $(n=1)$, Staphylococcus aureus $(n=2)$, Streptococcus constellatus $(n=2)$ (Figure 2). The subclassification of enteroviruses is shown in Figure 3. The incidence rates of epilepsy and neurodevelopmental outcomes for brain infections vs. controls populations were as follows: 7.5 vs. 0.3 (epilepsy), 2.7 vs. 2.0 (ADHD), 0.7 vs. 0 (ASD), 0.7 vs. 0.3 (epilepsy + ADHD), and 0.7 vs. 0.3 (ADHD + ASD). We did not find children with both epilepsy and ASD in the brain infections group and the control group.

\section{Neurodevelopmental Outcomes}

The possible risk factors during hospitalization associated with epilepsy and NDDs were illustrated in Figure 4. Intellectual disability and/or cerebral palsy account for the majority of longterm neurological sequelae throughout all possible prognostic markers. The incidence density rates of epilepsy were compared between the brain infections group and the control in analyses stratified by several variables, including age of CNS infection onset and associated pathogens. The overall risk of epilepsy in the brain infections group was higher than that in the control group (RR, 10.7; 95\% CI, 2.30-49.8; $p=0.002$ ). Moreover, in the stratified analysis, the risk of epilepsy in the brain infections group was higher than in the control group for all age ranges (except for children $>10$ years old) and for all different pathogens tested (Table 2).

\section{Incidence Rates and Relative Risks of ADHD}

Overall, no significant difference in the incidence rates and relatives risks of ADHD were noted between the brain infections group and the control group (RR, 1.51; 95\% CI, 0.53-4.27; $p=$ 0.56 ) as well as in stratified analyses (Table 3 ).

\section{Incidence Rates and Relative Risks of ASD}

Overall, no significant difference in the incidence rates and relative risks of ASD was noted between the brain infections group and the control group (RR, 4.7; 95\% CI, 0.42-51.7; $p=$ 0.20 ). However, notably, the incidence rates and relative risks of ASD were significantly higher in the children in the age interval of 2-5 years (RR, 21.3; 95\% CI, 1.33-341.4; $p=0.03$ ) (Table 4).

\section{DISCUSSION}

Although the identification of a pathogen known to cause encephalitis or meningitis confirms the diagnosis, in cases with negative CSF cultures, the diagnosis of viral encephalitis is made mostly based on clinical judgment when altered mental status and clinical presentation of viral encephalitis are suspected and abnormal CSF, electroencephalographic, or neuroimaging findings are obtained. Similarly, remarkable CSF results usually are sufficient to support the diagnosis of bacterial meningitis $(16,18)$.

However, identifying the causative pathogen in brain infections in children is crucial not only for treatment but also for neurodevelopmental prediction $(19,20)$. We focused on only three pathogenic organisms and found that Enterovirus accounted for the majority of brain infections, followed by GBS, S. pneumoniae, and HSV. Among the NDD studied the 


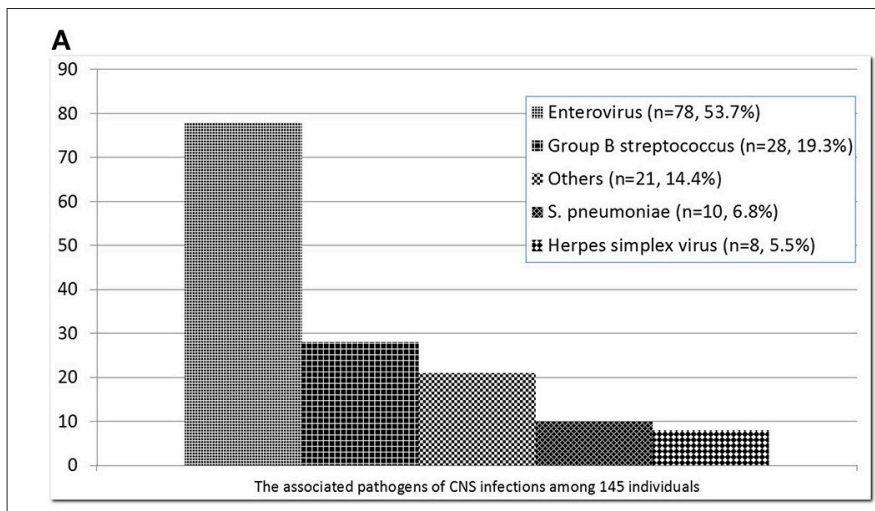

B

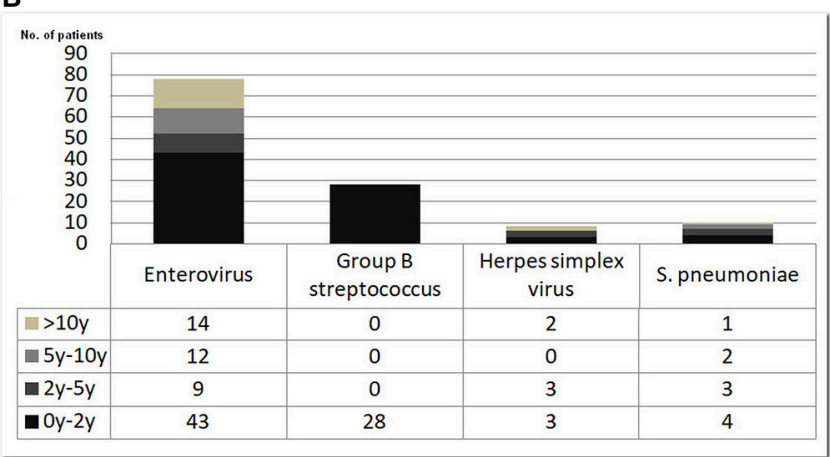

FIGURE 2 | Common pathogens of central nervous system infections among 145 affected subjects (A) and their stratification by age (B). The pathogenic organisms of "others" included Adenovirus ( $n=2)$, HHV-6 $(n=2), \operatorname{HHV}-7(n=1)$, Parvovirus $(n=1)$, Varicella-zoster virus $(n=1)$, Citrobacter koseri $(n=1)$, Enterobacter hormaechei $(n=1)$, Enterococcus casseliflavus $(n=1)$, Escherichia coli $(n=2)$, Haemophilus influenzae $(n=2)$, Neisseria meningitidis $(n=1)$, Listeria monocytogenes $(n=1)$, Mycobacteria tuberculosis complex $(n=1)$, Staphylococcus aureus $(n=2)$, Streptococcus constellatus $(n=2)$.

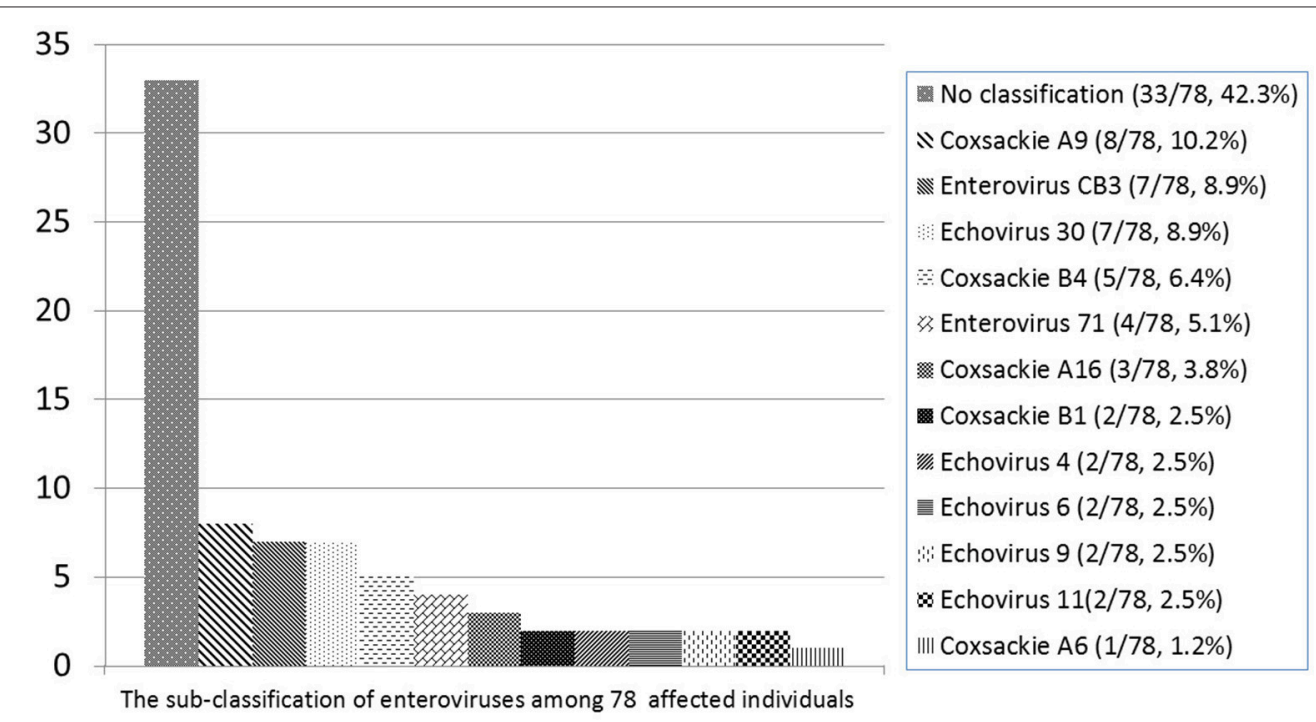

FIGURE 3 | Subclassification of enteroviruses among 78 affected individuals.

highest risk neurodevelopmental sequel was epilepsy among all pathogen classifications and most age intervals. Only children with enteroviral encephalitis during the preschool age interval had a higher risk of subsequent ASD.

Up to now, a small number of studies have explored the association between specific neurotropic pathogen infections during childhood and NDDs (15, 21-27). Among these studies, a study enrolled 86 children with viral culture-confirmed Enterovirus 71 (EV71) infection (15). That study found that those who had been infected with EV71 had a higher risk of developing ADHD-related symptoms. However, the study did not investigate the association of ADHD with other types of Enterovirus. According to Huang et al. (26), different type of Enterovirus infection might be responsible for different neurodevelopmental and behavior disorders (EV71 is associated with $\mathrm{ADHD}$, delayed neurodevelopment, and reduced cognitive functioning; coxsackievirus increases the risk of schizophrenia) $(1,27)$. Our study did not obtain the same results, possibly because only 4 out of 78 participants were identified with EV71 and a comprehensive group of enteroviruses was studied.

Unprovoked seizures and epilepsy can be long-term sequelae of bacterial meningitis and their risk were relatively higher for Streptococcus pneumoniae (28). Misra et al. reviewed acute symptomatic and late unprovoked seizures (epilepsy) in different viral encephalitides, which found: epilepsy caused by Herpes simplex encephalitis is associated with poor prognosis; Japanese encephalitis is the most common one associated with acute symptomatic seizures in children; Late-onset Nipah virus encephalitis is more commonly having seizures than acute encephalitis be (5). Although maternal and prenatal infections have been recognized to be associated with an increased risk of $\operatorname{ASD}(13,29)$, we propose that ASD may also be related to 


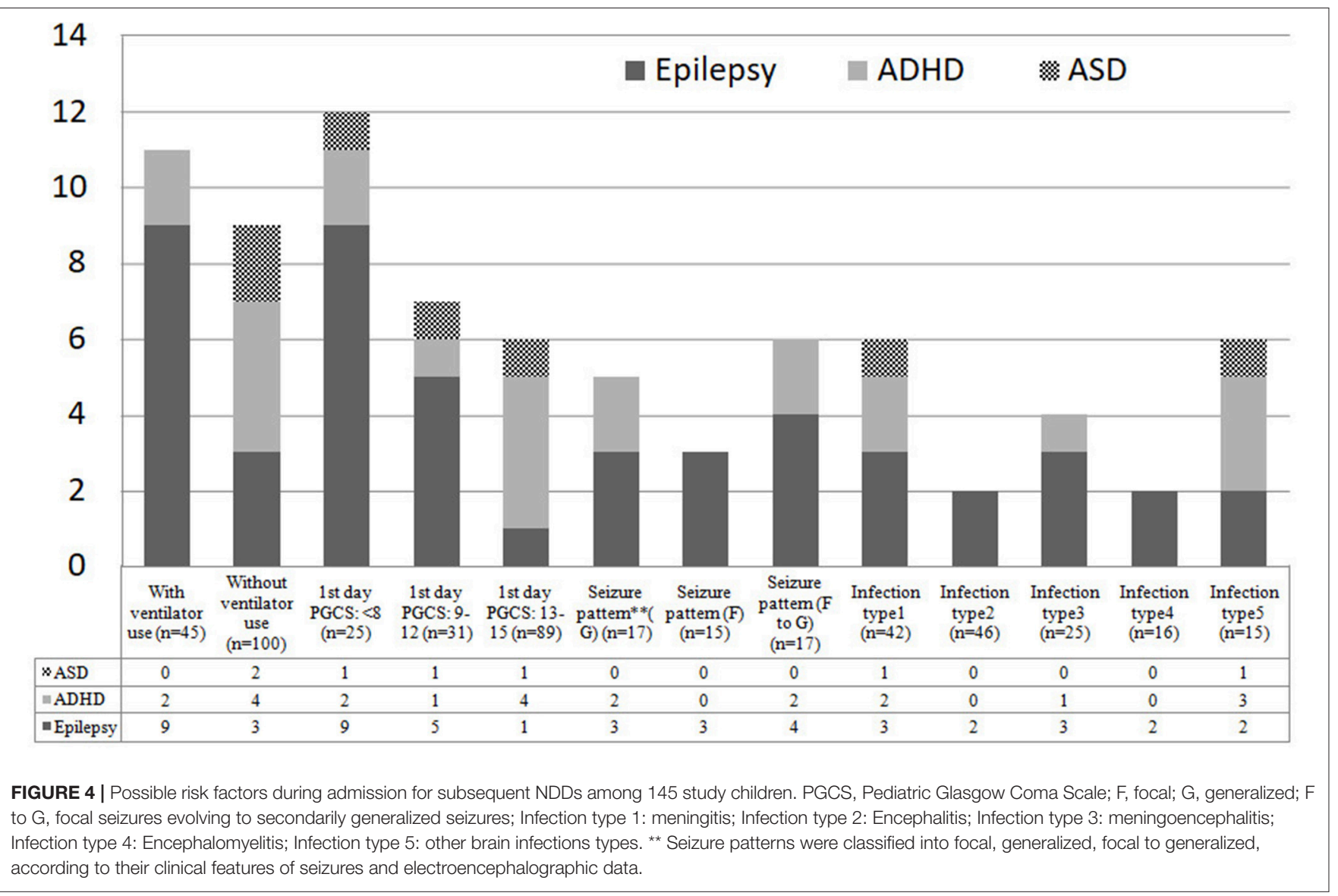

TABLE 2 | Incidence rates and relative risks of epilepsy for the controls compared with brain infections cohort.

\begin{tabular}{lccl}
\hline & Event & IR (\%) & RR (95\% Cl) \\
\hline Controls & 2 & 0.68 & Reference \\
All brain infections group & 12 & 8.27 & $10.7(2.30-49.8)^{\star \star}$ \\
Brain infections at (yr) & & & \\
$\quad 0-2$ & 9 & 6.20 & $12.3(2.56-59.69)^{\star \star}$ \\
$5-10$ & 1 & 0.68 & $16.20(2.21-118.38)^{\star \star}$ \\
$>10$ & 2 & 1.37 & $7.52(0.67-84.30)$ \\
Brain infections with different & & & \\
pathogens & & & \\
$\quad$ Enterovirus & 4 & 2.75 & $6.04(1.05-34.55)^{\star}$ \\
$\quad$ Herpes simplex virus & 1 & 0.68 & $22.3(2.00-249.93)^{\star \star}$ \\
$\quad$ Group B streptococcus & 2 & 1.37 & $9.90(1.35-72.30)^{\star}$ \\
$\quad$ S. pneumoniae & 1 & 0.68 & $14.70(1.50-68.40)^{\star}$ \\
$\quad$ Others & 4 & 2.75 & $6.04(1.05-34.55)^{\star}$ \\
\hline
\end{tabular}

$I R$, incidence rate; $R R$, risk ratio; yr, year; -, No event; N/A, not available.

${ }^{*} P<0.05$.

${ }^{* *} P<0.01$.

childhood enteroviral infection, especially during the preschool age interval. A potential explanation for this finding is that brain infections during these ages represent an exaggeration or abnormality of normal processes that occur during the
TABLE 3 | Incidence rates and relative risks of ADHD for the controls compared with brain infections cohort.

\begin{tabular}{lcclc}
\hline & Event & IR (\%) & RR (95\% Cl) & $\boldsymbol{p}$ \\
\hline Controls & 8 & 2.73 & Reference & \\
All brain infections group & 6 & 4.12 & $1.51(0.53-4.27)$ & 0.56 \\
$\begin{array}{l}\text { Brain infections at (yr) } \\
\quad-2\end{array}$ & 3 & 2.06 & $0.79(0.19-3.24)$ & 0.99 \\
$2-5$ & 2 & 1.37 & $5.93(0.90-38.9)$ & 0.99 \\
$\quad 5-10$ & 1 & 0.68 & $3.93(0.26-59.5)$ & 0.36 \\
Brain infections with different & & & & \\
pathogens & & & & \\
$\quad$ Enterovirus & 5 & 3.44 & $2.34(0.78-6.95)$ & 0.16 \\
$\quad$ Others & 1 & 0.68 & $1.52(0.198-11.6)$ & 0.51 \\
\hline
\end{tabular}

$I R$, incidence rate; RR, risk ratio; yr, year; -, No event; N/A: not available.

completion of brain development, which may impede children's communication and social skills. Hence, we presume infection older than preschool age in which brain development has approached completeness, may be affected to a lesser extent (which could also be observed in epilepsy group, Table 2). However, we are not clear why those infections younger than preschool age did not have the statistical difference in ASD incidence. More research with larger number of samples and more rigorous experimental design are necessary to explore 
TABLE 4 | Incidence rates and relative risks of ASD for the controls compared with brain infections cohort.

\begin{tabular}{lcclc}
\hline & Event & IR (\%) & RR (95\% Cl) & $\boldsymbol{p}$ \\
\hline $\begin{array}{l}\text { Controls } \\
\text { All brain infections group }\end{array}$ & 1 & 0.34 & Reference & \\
$\begin{array}{l}\text { Brain infections at (yr) } \\
\text { 0-2 }\end{array}$ & 1 & 0.68 & $3.77(0.23-60.3)$ & 0.34 \\
$2-5$ & 1 & 0.68 & $21.3(1.33-341.4)^{\star}$ & 0.03 \\
$\begin{array}{l}\text { Brain infections with different } \\
\text { pathogens }\end{array}$ & & & & 0.20 \\
$\quad$ Enterovirus & 2 & 1.37 & $8.06(0.73-88.9)$ & 0.08 \\
\hline
\end{tabular}

$I R$, incidence rate; $R R$, risk ratio; $y r$, year; -, No event; N/A, not available. ${ }^{\star} P<0.05$.

this issue. We also found that a small subset of patients had dual neurodevelopmental comorbidities (i.g. epilepsy+ASD or $\mathrm{ADHD}+\mathrm{ASD}$ ). Although the case number was too small to further analyze, recent studies has shown that NDDs have been considered to have high rates of both homotypic and heterotypic comorbidity, which needs to be taken into consideration in health service planning and treatment delivery $(30,31)$.

Other risk factors possibly associated with subsequent NDDs during admission were also identified in this study, including ventilator use, low Glasgow Coma Scale score in the first day, different infection types and patterns of seizure (Figure 4). Remarkably, the more critical the patients initially presented, the higher risk of subsequent NDDs they may have. Furthermore, additional research focusing on neuroimaging, genetic susceptibility, or proinflammatory cytokines and other environmental exposures may identify potential mechanisms underlying NDDs in children with different patterns of brain infections.

Our study had several limitations. First, as stated above, the causal role of pathogenic agents is sometimes difficult to determine from human CSF (e.g., influenza virus and cytomegalovirus), and these types of infection may have inevitably been lost under the present study design, which rendered us unable to obtain information on holistic neurological outcomes for all etiological brain infections. Second, several non-infectious causes, such as patient's immune status, neurogenetic factors, and structural or metabolic disorders, may be confounders for the development of epilepsy and NDDs in childhood and were not investigated in the present study. Third, because this was a retrospective longitudinal study that used patients' medical records obtained from a single medical center, patients who opted to receive medical care at another hospital or clinic were not included in this study, which may have affected the results. Fourth, RR for ASD (in children who had brain infection between 2 and 5 year age) was based on only one patient. In addition, only certain pathogens and NDDs were focused on. Therefore, more samples and diverse pathogens as well as types of NDDs should be included in the future research to support our finding.

In conclusion, our study identified common etiological causes of brain infections in Taiwanese children. Among them, Enterovirus accounted for the majority of infections, followed by GBS, S. pneumoniae, and HSV. In addition to ID, which is considered the most common neurological sequelae in brain infections, our study further shows that the highest-risk neurodevelopmental sequelae was epilepsy among all pathogen classifications and most age intervals. Children who had a diagnosis of brain infections (specially Enterovirus) should be followed since they are at greater risk of developing epilepsy and ASD. Only children with enteroviral encephalitis during the preschool age interval had a higher risk of ASD. Therefore, early assessment and identification of epilepsy and other NDDs facilitate early intervention and treatment among these children. Additional studies should examine the relationship between additional etiological sources and their possible associations with other NDDs, such as Tourette syndrome, communication disorders, and speech and language disorders.

\section{ETHICS STATEMENT}

After a full description of the study, written informed consent of participation was obtained from the legal guardians. The study protocol was approved by the Ethics Review Board of the China Medical University ethics committee (Approval \# CMUH108-REC1-023 and \# CMUH107-REC2-017 and \#CRS106-027 and \#CRS-106-031).

\section{AUTHOR CONTRIBUTIONS}

S-YH collected and analyzed the data and prepared the draft. C-HL and W-DL participated in the design of the study and wrote the manuscript. I-CL and I-CC compiled the statistics of this study and participated in the editing and revising the tables. All authors read and approved the final manuscript.

\section{ACKNOWLEDGMENTS}

We would like to thank the Genetic Medicine Laboratory of China Medical University Hospital, Ministry of Science and Technology of ROC (MOST 107-2314-B-039-003) and China Medical University Hospital Medical Research Department (DMR-108-199) for giving support and assistance to this work.

\section{REFERENCES}

1. Chang LY, Huang LM, Gau SS, Wu YY, Hsia SH, Fan TY, et al. Neurodevelopment and cognition in children after enterovirus 71 infection. N Engl J Med. (2007) 356:1226-34. doi: 10.1056/NEJMoa065954

2. Annegers JF, Hauser WA, Beghi E, Nicolosi A, Kurland LT. The risk of unprovoked seizures after encephalitis and meningitis. Neurology. (1988) 38:1407-10.

3. Arditi M, Mason EO Jr, Bradley JS, Tan TQ, Barson WJ, Schutze GE, et al. Three-year multicenter surveillance of pneumococcal meningitis in children: 
clinical characteristics, and outcome related to penicillin susceptibility and dexamethasone use. Pediatrics. (1998) 102:1087-97.

4. Pomeroy SL, Holmes SJ, Dodge PR, Feigin RD. Seizures and other neurologic sequelae of bacterial meningitis in children. N Engl J Med. (1990) 323:1651-7.

5. Misra UK, Tan CT, Kalita J. Viral encephalitis and epilepsy. Epilepsia. (2008) 49 (Suppl 6):13-8. doi: 10.1111/j.1528-1167.2008.01751.x

6. Mullin AP, Gokhale A, Moreno-De-Luca A, Sanyal S, Waddington JL, Faundez V. Neurodevelopmental disorders: mechanisms and boundary definitions from genomes, interactomes and proteomes. Transl Psychiatry. (2013) 3:e329. doi: 10.1038/tp.2013.108

7. Watts TJ. The pathogenesis of autism. Clin Med Pathol. (2008) 1:99-103. doi: $10.4137 /$ CPath.S1143

8. Karimi P, Kamali E, Mousavi SM, Karahmadi M. Environmental factors influencing the risk of autism. J Res Med Sci. (2017) 22:27. doi: 10.4103/1735-1995.200272

9. Cordeiro CN, Tsimis M, Burd I. Infections and brain development. Obstet Gynecol Surv. (2015) 70:644-55. doi: 10.1097/OGX.0000000000000236

10. Koontz T, Bralic M, Tomac J, Pernjak-Pugel E, Bantug G, Jonjic S, et al. Altered development of the brain after focal herpesvirus infection of the central nervous system. J Exp Med. (2008) 205:423-35. doi: 10.1084/jem.20071489

11. Dorsett M, Liang SY. Diagnosis and treatment of central nervous system infections in the emergency department. Emerg Med Clin North Am. (2016) 34:917-42. doi: 10.1016/j.emc.2016.06.013

12. Muhle RA, Reed HE, Stratigos KA, Veenstra-VanderWeele J. The emerging clinical neuroscience of autism spectrum disorder: a review. JAMA Psychiatry. (2018) 75:514-23. doi: 10.1001/jamapsychiatry.2017.4685

13. Mazina V, Gerdts J, Trinh S, Ankenman K, Ward T, Dennis MY, et al. Epigenetics of autism-related impairment: copy number variation and maternal infection. J Dev Behav Pediatr. (2015) 36:61-7. doi: 10.1097/DBP.0000000000000126

14. Singh G, Prabhakar S. The association between central nervous system (CNS) infections and epilepsy: epidemiological approaches and microbiological and epileptological perspectives. Epilepsia. (2008) 49 (Suppl 6):2-7. doi: 10.1111/j.1528-1167.2008.01749.x

15. Gau SS, Chang LY, Huang LM, Fan TY, Wu YY, Lin TY. Attentiondeficit/hyperactivity-related symptoms among children with enterovirus 71 infection of the central nervous system. Pediatrics. (2008) 122:e452-8. doi: 10.1542/peds.2007-3799

16. Venkatesan A, Tunkel AR, Bloch KC, Lauring AS, Sejvar J, Bitnun A, et al. Case definitions, diagnostic algorithms, and priorities in encephalitis: consensus statement of the international encephalitis consortium. Clin Infect Dis. (2013) 57:1114-28. doi: 10.1093/cid/cit458

17. Leber AL, Everhart K, Balada-Llasat JM, Cullison J, Daly J, Holt S, et al. Multicenter evaluation of biofire filmarray meningitis/encephalitis panel for detection of bacteria, viruses, and yeast in cerebrospinal fluid specimens. J Clin Microbiol. (2016) 54:2251-61. doi: 10.1128/JCM.00730-16

18. Blazer S, Berant M, Alon U. Bacterial meningitis. Effect of antibiotic treatment on cerebrospinal fluid. Am J Clin Pathol. (1983) 80:386-7.

19. Kim MA, Park KM, Kim SE, Oh MK. Acute symptomatic seizures in CNS infection. Eur J Neurol. (2008) 15:38-41. doi: 10.1111/j.1468-1331.2007.01996.x
20. Soni V, Singhi P, Saini AG, Malhi P, Ratho RK, Mishra B, et al. Clinical profile and neurodevelopmental outcome of new-onset acute symptomatic seizures in children. Seizure. (2017) 50:130-6. doi: 10.1016/j.seizure.2017. 06.013

21. Chou IC, Lin CC, Kao CH. Enterovirus encephalitis increases the risk of attention deficit hyperactivity disorder: a taiwanese populationbased case-control study. Medicine (Baltimore). (2015) 94:e707. doi: 10.1097/MD.0000000000000707

22. Hadzic E, Sinanovic O, Memisevic H. Is bacterial meningitis a risk factor for developing attention deficit hyperactivity disorder. Isr J Psychiatry Relat Sci. (2017) 54:54-57.

23. Spann MN, Sourander A, Surcel HM, Hinkka-Yli-Salomäki S, Brown AS Prenatal toxoplasmosis antibody and childhood autism. Autism Res. (2017) 10:769-777. doi: 10.1002/aur.1722

24. Teixeira AL, Barichello T. Psychiatric syndromes secondary to central nervous system infection. Braz J Psychiatr. (2012) 34:221. doi: 10.1590/S1516-44462012000200017

25. Wang C, Geng H, Liu W, Zhang G. Prenatal, perinatal, and postnatal factors associated with autism: a meta-analysis. Medicine (Baltimore). (2017) 96:e6696. doi: 10.1097/MD.0000000000006696

26. Huang H, Shih SR. Neurotropic enterovirus infections in the central nervous system. Viruses. (2015) 7:6051-66. doi: 10.3390/v7112920

27. Euscher E, Davis J, Holzman I, Nuovo GJ. Coxsackie virus infection of the placenta associated with neurodevelopmental delays in the newborn. Obstet Gynecol. (2001) 98:1019-26. doi: 10.1016/S0029-7844(01) 01625-8

28. Murthy JM, Prabhakar S. Bacterial meningitis and epilepsy. Epilepsia. (2008) 49 Suppl 6:8-12. doi: 10.1111/j.1528-1167.2008.01750.x

29. Johnson CP, Myers SM. American academy of pediatrics council on children with disabilities. Identification and evaluation of children with autism spectrum disorders. Pediatrics. (2007) 120:1183-215. doi: 10.1542/peds.2007-2361

30. Hansen BH, Oerbeck B, Skirbekk B, Petrovski BÉ, Kristensen H. Neurodevelopmental disorders: prevalence and comorbidity in children referred to mental health services. Nord J Psychiatry. (2018)72:285-291. doi: 10.1080/08039488.2018.1444087

31. Lewis ML, Kesler M, Candy SA, Rho JM, Pittman QJ. Comorbid epilepsy in autism spectrum disorder: Implications of postnatal inflammation for brain excitability. Epilepsia. (2018) 59:1316-1326. doi: 10.1111/epi.14440

Conflict of Interest Statement: The authors declare that the research was conducted in the absence of any commercial or financial relationships that could be construed as a potential conflict of interest.

Copyright (c) 2019 Lin, Lin, Chou, Lee and Hong. This is an open-access article distributed under the terms of the Creative Commons Attribution License (CC BY). The use, distribution or reproduction in other forums is permitted, provided the original author(s) and the copyright owner(s) are credited and that the original publication in this journal is cited, in accordance with accepted academic practice. No use, distribution or reproduction is permitted which does not comply with these terms. 